\title{
Der Integrierte Katalog der Mediathek der Hochschule für Gestaltung und Kunst Basel
}

\author{
Katalogkompetenz und Kommunikation im digitalen Wandel
}

https://doi.org/10.1515/bfp-2018-0002

Zusammenfassung: Der „integrierte Katalog“ der Mediathek der Hochschule für Gestaltung und Kunst (HGK) FHNW Basel versammelt die wichtigsten Informationsquellen der HGK und versucht, mithilfe von sinnstiftenden Daten-Layouts Informationskompetenz zu vermitteln. Im Folgenden werden Motivation und Hintergründe erläutert, da sich an der Oberfläche (Interface) die mediatheksseitigen Interessen und die nutzerseitigen Anforderungen und Recherchekonventionen treffen. Sie sind Bestandteil der fließenden künstlerischen, gestalterischen und wissenschaftlichen Kommunikation.

Schlüsselwörter: Katalog; Data Curation; Informationskompetenz

The Integrated Catalogue of the Media Library at Academy of Art and Design Basel

Catalogue Competence and Communication in the Digital Age

Abstract: The "integrated catalogue" of HGK combines the most important information resources of the Academy of Art and Design HGK FHNW Basel. By using meaningful data layouts, we try to convey information competences. The following article describes our motivation and provides background information for the catalogue's development. Since interests of the media library encounter with user requirements and their research conventions at the catalogues surface, this interface is part of the flowing artistic, creative and scientific communication.

Keywords: Catalogue; data curation; information literacy

*Kontaktperson: Tabea Lurk, tabea.lurk@fhnw.ch Jürgen Enge, juergen.enge@fhnw.ch
Inhalt

1 Ausgangslage................. 57

2 Die Mediathek und ihr Informationsangebot im

Kontext .................... 59

2.1 Teilbestände und Spezialsammlungen . . . . . . 60

2.2 Workflows und Prozesse. . . . . . . . . . 63

3 Katalogkompetenz................. 64

3.1 Usability \& Content-Design . . . . . . . . . . . . 64

3.2 Werkzeuge .................. 66

4 Fazit und Ausblick . . . . . . . . . . . . . . . . . . 67

\section{Ausgangslage}

Die meisten Hochschulen sind heute damit konfrontiert, dass der vielfältige Mix an Wissensressourcen, den sie für unterschiedliche Nutzergruppen zur Verfügung stellen sollen und wollen, über verschiedenste Datenbasen verteilt sind. Selten sind alle lizensierten E-Books, E-Journals und Datenbanken im gleichen Katalog referenziert, wie die analogen (Leih-)Medien, die Spezialsammlungen, die Forschungsdaten und die Open-Access-Ressourcen. Für die Schweiz ist das Wissensportal der ETH zu erwähnen, welches eine Suche über ganz unterschiedliche Teilbestände der ETH-Sammlungen und -Archive ermöglicht - inklusive klassischer Bibliotheksbestände. Häufig kommen aber weitere, mitunter digital erschlossene oder digitalisierte Spezialsammlungen hinzu, die zwar z.T. frei im Internet zur Verfügung stehen, aber eher selten so in die eigene Plattform eingebunden sind, dass sie direkt und gemeinsam mit den übrigen Beständen durchsucht werden können. ${ }^{1}$ Hannah Bennett spricht mit Blick auf ihr eigenes

1 Dass Anbieter digitaler Ressourcen wie z. B. EBSCO immer häufiger die Einbindung von Suchschlitzen (widgets) auf externen Webseiten erlauben, um die Nutzer in die eigene Suchumgebung zu integrieren 


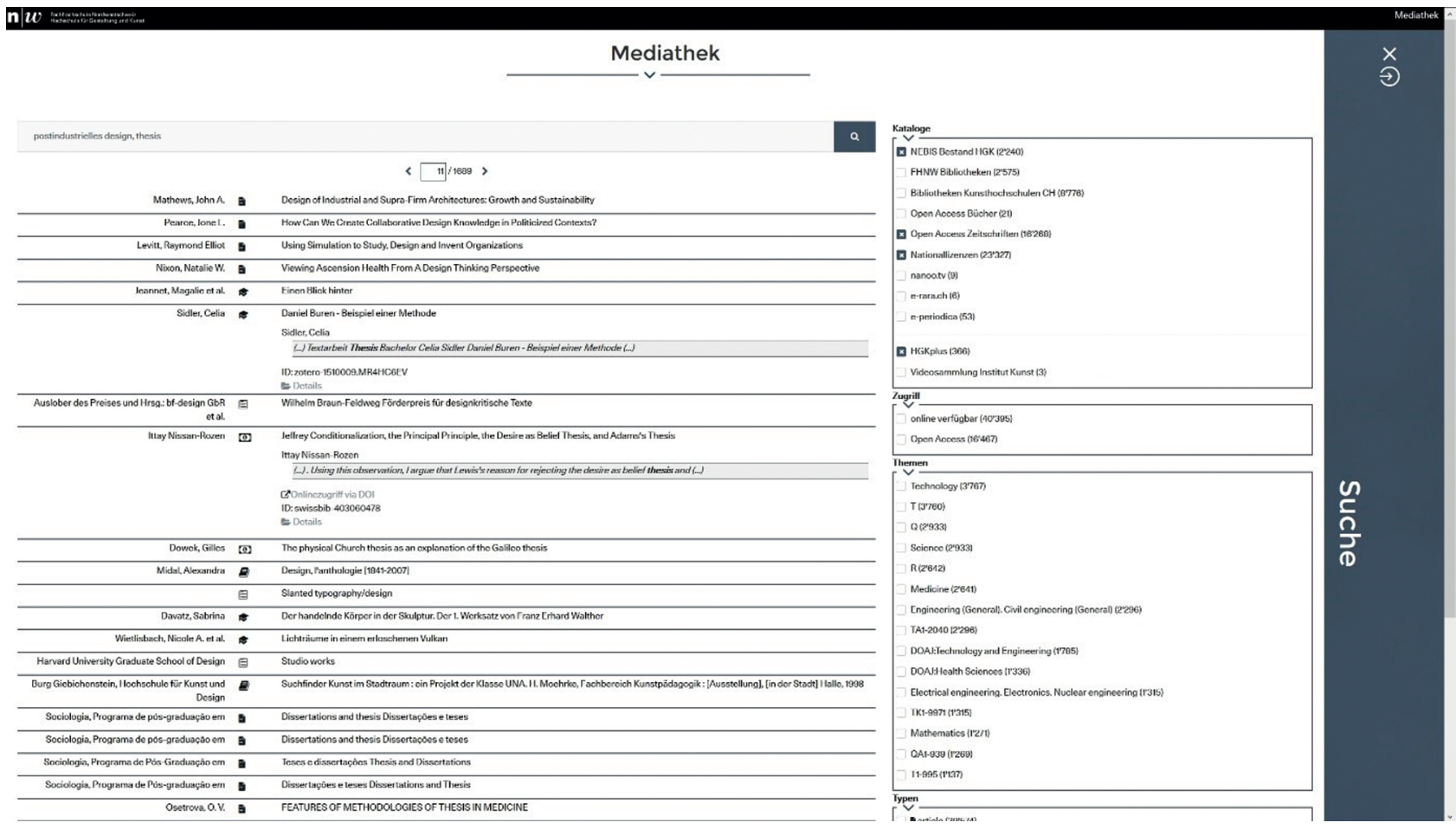

Abb. 1: Listenansicht des integrierten Katalogs. In der Trefferliste finden sich neben Büchern auch eine Abschlussarbeit, eine CD-ROM und ein Aufsatz, was die Icons in der Mitte anzeigen. Die rechte Spalte weist die Kataloge und Spezialsammlungen aus.

Arbeitsumfeld von „exit research strategies“, um Rechercheformen zu charakterisieren, die „focusing on free resources beyond Yale's vast electronic and print holdings". ${ }^{2}$

Zwar kann man argumentieren, dass die Kenntnis dieser externen Plattformen zu jenen Kompetenzen gehört, die durch das Studium erworben werden oder dass externe Inhalte sowieso nicht aufgenommen werden, weil keine Qualitätsgarantie gewährleistet werden kann (Policy-Problem). Dennoch sollten die Wege zu den verteilten Ressourcen insgesamt abgekürzt werden und zwar unabhängig davon, wohin die digitalen Wege zeigen. Zudem praktizieren die großen „Informationsmaschinen“ und -mechanismen, die den Wissenschaftsbetrieb heute nicht nur mit Daten versorgen, sondern auch regulieren (Lizenzen) und strukturieren (Paketierung, Disvocery-Mechanismen), eine Form der Optimierung, die den Ansprüchen einer Kunstund Gestaltungshochschule eher selten Genüge leistet. In erster Linie geht es uns darum, künstlerisch gestalterische Kreativität und Souveränität zu ermöglichen und ein spartenübergreifendes Denken zu forcieren. Systemfehler werden als spannend betrachtet und häufig variieren nicht nur

(und damit das Nutzungsverhalten zu studieren), wirkt auf uns in diesem Zusammenhang unzureichend.

2 Bennett (2006) 39. die Recherchekontexte und Erfahrungshorizonte, ${ }^{3}$ sondern auch die Werte und Methoden. In diesem Zusammenhang spricht das Inhaltsverzeichnis der Anthologie Facing value von Maaike Lauwaerts und Francien van Westrenens Bände, denn die Überschriften der neun Kapitel, die ja Wertvorstellungen in den Künsten zum Thema haben, lesen sich wie Handlungsanweisungen: „be unproductive“, „,hesitate \& question“, „share“, „improvise“, „,invite \& participate“, „embrace the void“, „play“, „support“ und „unite“. ${ }^{4}$ So sind wir der Meinung, dass es der Informationskompetenz zuträglich ist, wenn Verweise auf externe Datenbasen und Quellen gegeben werden, da diese den Reflexionshorizont erweitern und die Neugierde wecken.

Daher wurde in der Hochschule für Gestaltung und Kunst Basel (HGK) im Frühjahr 2016 mit dem Aufbau eines „integrierten Katalogs“ begonnen. ${ }^{5}$ Der Katalog liefert ne-

3 Walczak et al. (2010) 194 haben darauf hingewiesen, dass zum einen die Lehrenden an Kunstakademien zumeist einen künstlerischgestalterischen Hintergrund haben und entsprechend weniger jene Rechercheerfahrungen aufweisen, die in anderen Disziplinen unter dem Vorzeichen der wissenschaftlichen Forschung von Generation zu Generation weitergegeben werden. Zum anderen variiert auch die Struktur und Zusammensetzung der Abschlüsse.

4 Lauwaerts und van Westrenens (2017).

5 Zur Projektentwicklung vgl. https://mediathek.hgk.fhnw.ch/word press/technische-beschreibung/projektverlauf/. Alle Internetadressen wurden am 29.09.2017 geprüft. 
ben dem Verweis auf analoge Medienbestände eine HGKzentrierte (customized) Rechercheinfrastruktur, die hauseigene und externe Medienbestände einbezieht, die erweiterbar ist und versucht, eine neue Haltung zu Recherchekontexten sowie Informations- und Katalogkompetenz zu implementieren. Zur klassischen Listenansicht (reine Liste) und der Listenvorschau (aufgeklappte Einzeldatensätze der Listenansicht, vgl. Abb. 1) kommen kuratierte Detailseiten, welche die Ergebnisse in semantische Zusammenhänge einbetten. ${ }^{6}$

\section{Die Mediathek und ihr Informationsangebot im Kontext}

Die HGK ist ein Departement der Fachhochschule Nordwestschweiz (FHNW), die von den Kantonen Aargau, Basel-Stadt, Basel-Landschaft und Solothurn getragen wird. Die Bereichsbibliotheken der einzelnen FHNW-Hochschulen und -Standorte gehören dem Netzwerk von Bibliotheken und Informationsstellen in der Schweiz (NEBIS) sowie die Musikhochschulen FHNW dem Informationsverbund (IDS) Basel Bern an. Für die Verfügbarkeit von lizensierten e-Ressourcen bedeutet dies, dass e-Medien, die in einem FHNW-Departement lizensiert wurden, i.d. R. auch für die anderen Departemente zur Verfügung stehen. Da die bibliographischen Daten aus der swissbib stammen, die seit 2009 die Daten von ca. 960 Schweizer Bibliotheken aggregiert, ${ }^{7}$ kann der FHNW-Medienbestand als virtuelle Sammlung verbundsübergreifend ausgewiesen werden. Auch andere digitale Spezialsammlungen der Schweiz wie ePeriodica, ${ }^{8}$ e-Rara ${ }^{9}$ oder das von der Zürcher Hochschule der Künste (ZHdK) und dem Schweizer EDU-Netzanbieter Switch für die Fachhochschulen freigegebene Nanoo-TV ${ }^{10}$ sind im integrierten Katalog als virtuelle Sammlungen ausweisen (Abb. 1, rechte Spalte).

Wie alle Fachhochschulen der Schweiz profitiert die HGK zudem von Konsortialpaketen, die für das Konsortium der Schweizer Fachhochschulen aus Mitteln der Swissuniversities finanziert werden. Während ein kleiner

6 Der Katalog ist online einsehbar unter https://mediathek.hgk. fhnw.ch.

7 Hipler (2017) 8.

8 Mit e-Periodica stellt die ETH „Schweizer Zeitschriften online“ zur Verfügung, vgl. https://www.e-periodica.ch.

9 E-rara.ch macht seltene, digitalisierte Drucke aus Schweizer Bibliotheken zugänglich, vgl. http://www.e-rara.ch.

10 Nanoo.tv EDU ermöglicht die Nutzung von Film- und Fernsehsendungen an Schulen und anderen Bildungseinrichtungen, https://por tal.nanoo.tv. Zum Projekt vgl. MIZ (2017).
Teil über die NEBIS-Bestände ausgewiesen wird, sind die Zeitschriftenpakete über die Elektronische Zeitschriftendatenbank der Universität Regensburg (EZB) ausgewiesen, deren Listen turnusmäßig in unser System eingespeist werden. Sie sind aus Gründen der besseren Nutzbarkeit bei der Standardsuche (NEBIS Bestand HGK) eingebunden. Hinzu kommen die Nationallizenzen, für die eine eigene Sammlung angelegt wurde, um das Bewusstsein für diesen noch jungen Schatz zu stärken. Nicht zuletzt werden frei verfügbare Open-Access-Publikationen ausgewiesen, die derzeit noch als zwei Teilsammlungen angezeigt werden, da es ein strategisches Ziel der Mediathek ist, das Bewusstsein für Open Access zu fördern. ${ }^{11}$ Diese Inhalte stehen auch Nutzergruppen frei zur Verfügung, die keine Schweizer Hochschulaffiliierung (mehr) haben, wie unsere Absolvierenden (Alumni) oder die interessierte Öffentlichkeit.

Eine möglichst umfassende Angebotsbreite ist für die HGK wichtig, da das Studienangebot von Mode, Industrie und dem postindustriellen Design, über die visuelle und die integrative Gestaltung bis zu Kunst, Innenarchitektur und Szenografie sowie Kunstvermittlung reicht und die zugehörigen Theoriebereiche, inklusive der Ästhetischen Praxis oder den Design- und Medienkulturen, umfasst. Allein im Industrial Design sollte die Recherchetiefe neben Grundlagen der Materialkunde, -wissenschaft und -technologie auch (Kontext-)Informationen zu spezifischen medizinischen Anforderungen (z.B. für die Gestaltung von medizinischen Kleinstgeräten), Sicherheitsstandards und -konventionen (z.B. für Sport- oder Sicherheitskleidung und Werkzeuge) bieten und elektronische, computerbasierte oder automotive Aspekten abdecken.

Die weitläufigen Informationsbedürfnisse werden teilweise durch den NEBIS-Fernleih-Verbund abgefedert, dem die Mediathek angehört. Allerdings ist dort wiederum nur ein kleiner Teil der Industrial-Design-Abschlussarbeiten der HGK referenziert. Die übrigen Thesen, Inhalte, Produktionen sowie weitere Ressourcen und Bestände der HGK finden sich an unterschiedlichsten (Speicher-)Orten, teilweise in historisch gewachsenen Datenbanken und spora-

11 Neben den Inhalten aus dem Directory of Open Access Books (DOAB) und dem Directory of Open Access Journals (DOAJ inkl. Artikeln) werden derzeit die Open-Access-Publikationslinien von De Gruyter und Springer direkt indexiert. Aufgrund der wachsenden Anzahl an (neuen) Teilsammlungen wird die Menüstruktur am rechten Rand der Webseite immer wieder leicht überarbeitet. Das Grundkonzept, dass oben „virtuelle“ Sammlungen, also Daten-Zusammenstellungen aus unterschiedlichen Quellen, und im unteren Bereich der Katalogbox ungefilterte Datenquellen angezeigt werden, wurde bisher beibehalten. 


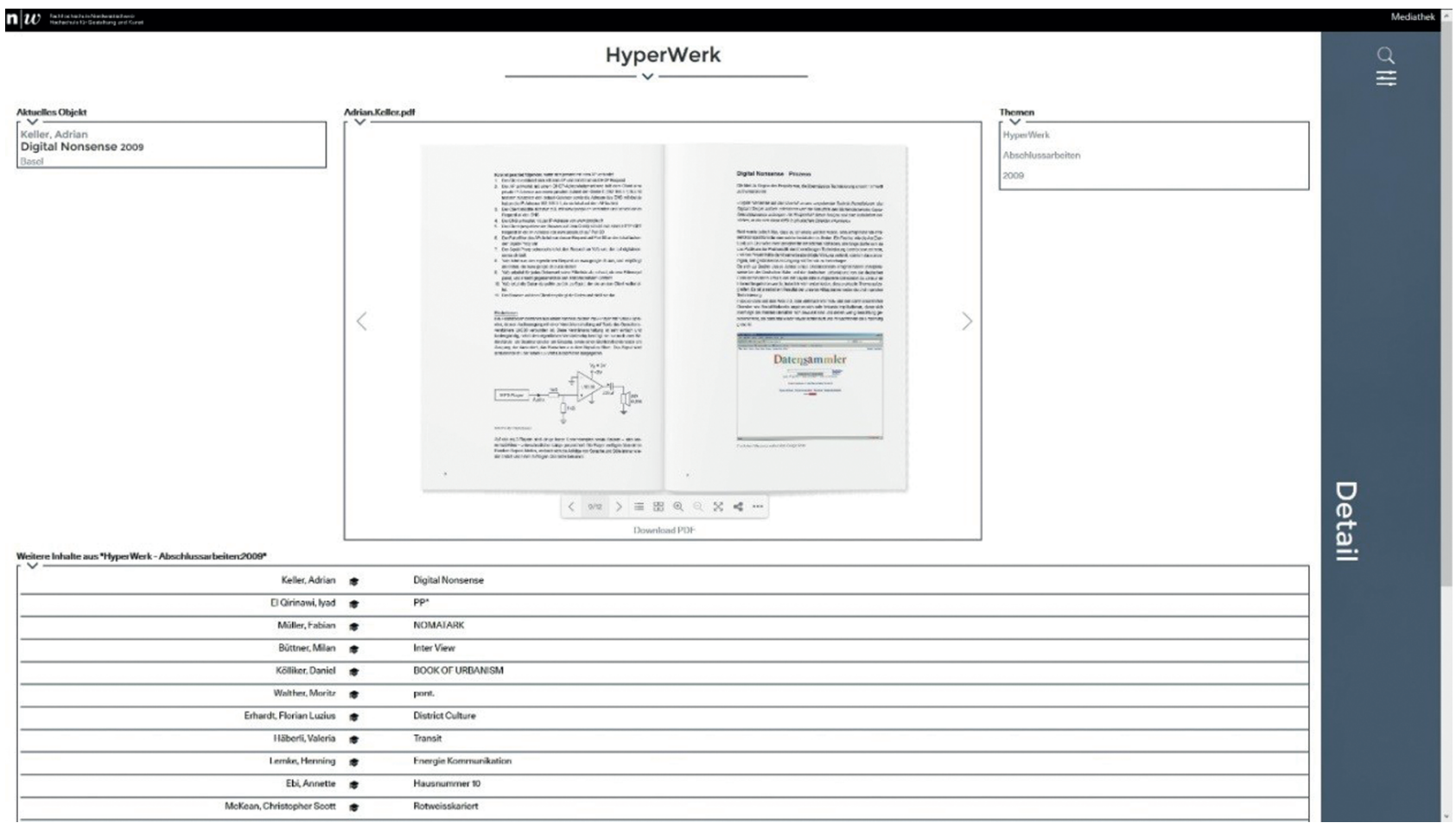

Abb. 2: Detailansicht mit Abschlussarbeiten des Instituts Hyperwerk aus dem Jahr 2009

disch auch als Metadateneintrag im zentralen Forschungsrepositorium der FHNW (IRF). ${ }^{12}$

Diese Verteilung hat z.T. gute Gründe, denn bestimmte Inhalte erreichen gar keinen „Publikationsstatus“ oder gehören aus anderen Gründen nicht in den Bibliotheksverbund. Dennoch sollten sie im Hochschulkontext auffindbar sein, zumal bei künstlerisch-gestalterischen Arbeiten der Wert mit der Zeit eher steigt, als dass die Ergebnisse veralten. Daher haben viele Kunsthochschulen mit dem Aufbau entsprechender Archive begonnen und auch die Mediathek versucht - wie der folgende Überblick über einige Teilbestände verdeutlicht - mithilfe von memorierenden Workflows die hauseigene Erinnerungskultur zu unterstützen.

\subsection{Teilbestände und Spezialsammlungen}

Vereinfacht lassen sich die nicht via NEBIS ausgewiesenen Teilbeständen und Sammlungen der Mediathek HGK in drei große Bereiche untergliedern: 1) HGK-Materialien \& Dokumentationen, 2) Lehrmittel \& Kooperationen, 3) Schenkungen \& Capta. ${ }^{13}$ Es liegt in der Natur einer Kunsthochschule,

12 Das noch im Aufbau befindliche Repository der FHNW findet sich online unter http://irf.fhnw.ch/.

13 „Capta are units that have been selected and harvested from the sum of all potential data, where data are the total sum of facts in relation dass die Trennlinien mitunter unscharf sind und dass die Herausforderung durch die Vielzahl der unterschiedlichen Medienformate und Dokumentationskonventionen all jener Akteure wächst, die sich für die Erhaltung und Überlieferung der (Meta-)Daten eingesetzt haben. ${ }^{14}$

Unter den HGK-Materialien \& Dokumentationen können folgende Konvolute hervorgehoben werden:

- Das Digitale Archiv des Instituts Industrial Design, das zwischen 2003 und 2017 in einer MySQL-Datenbank gepflegt wurde und neben der Aufbewahrung von Modul-, Seminar- und Projektarbeiten vor allem die institutsinterne Dokumentation gewährleistete. Harmonisierte Schlagwörter, Kategorien und eine klare Aufnahmepolicy, welche z.B. auch die Bildqualität vorgeschrieben hat, haben zur systematischen Erscheinungsweise beigetragen. 2016 ist das bisherige System temporär ausgefallen. Bei der Migration der Daten in den integrierten Katalog sind 2017 ferner Daten des damals ebenfalls noch in Aarau angesiedelten Studiengangs Medienkunst zum Vorschein gekommen, die demnächst aufbereitet werden.

to an entity; in other words, with respect to a person, data is everything that it is possible to know about that person, capta is what is selectively captured through measurement“, Kitchin und Dodge(2011) 5.

14 Ihnen, die so viel Vorarbeit geleistet haben, gilt unser besonderer Dank. 


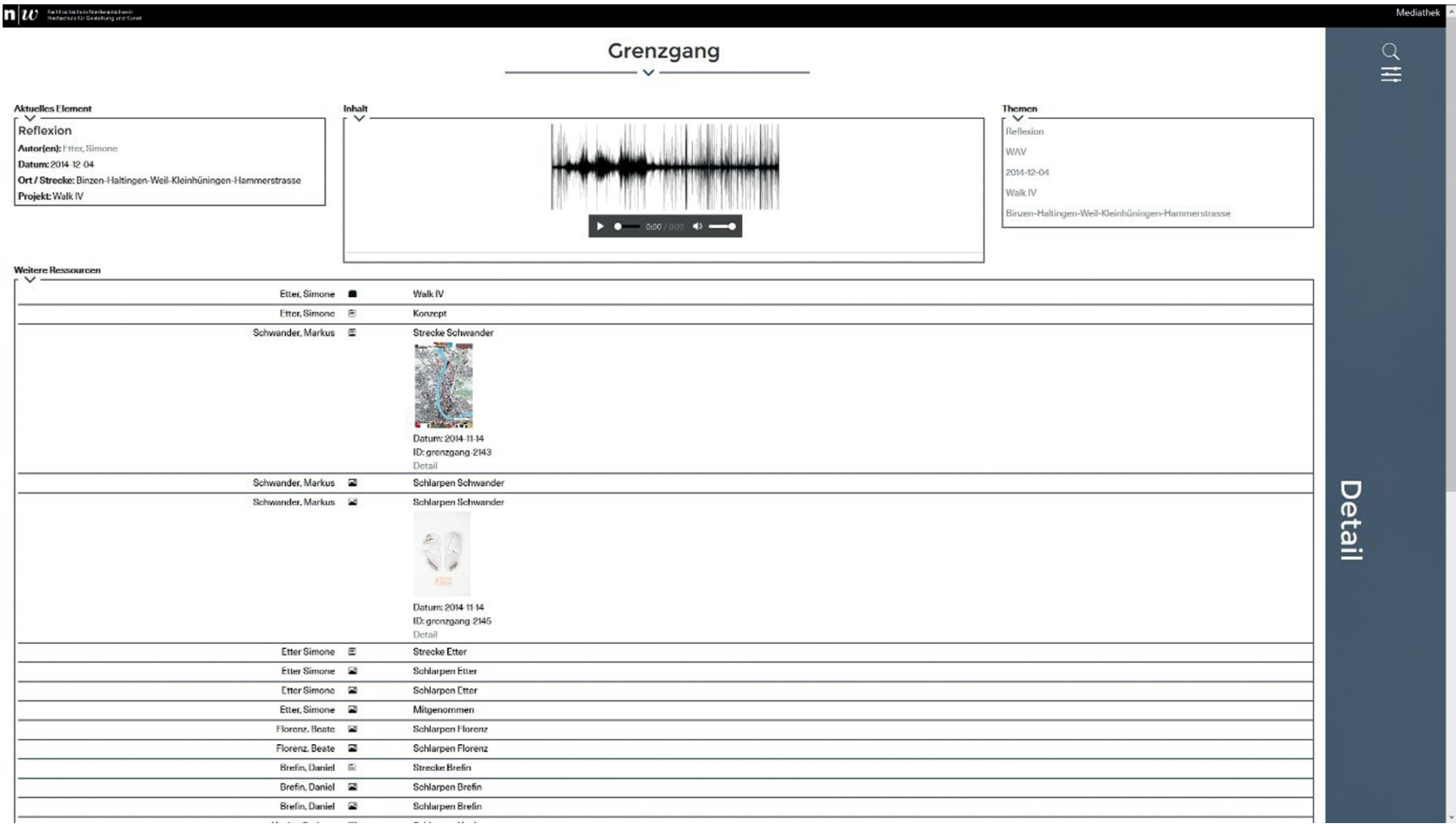

Abb. 3: Detailansicht Grenzgang, welche einen kurzen Einblick zur Heterogenität der Materialien gibt.

- Die Diplom- und Thesensammlungen der Institute werden seit dem Sommer 2017 in der Mediathek erschlossen. Bisher wurden 278 Abschlussarbeiten des Instituts Hyperwerk aus den Jahren 2002-2016 (ohne 2007) retro-katalogisiert, die online einsehbar sind. Vom Institut Kunst wurden 706 Text- und Theoriearbeiten erfasst, die bis ins Jahr 1995 zurückreichen und teilweise analog in der Mediathek einsehbar sind. 387 Dokumente aus den Jahre 2007 bis 2017 liegen digital vor, werden aber aufgrund von Persönlichkeitsund Urheberrechten nur institutsintern angezeigt. ${ }^{15}$ Ebenfalls 2017 wurde ein neuer Workflow zur Datenerfassung für die DiplomHGK-Webseite implementiert, sodass diese Ressource und später dann auch die Diplomarbeiten direkt über den integrierten Katalog verwaltet werden können.

- Zu den Ressourcen aus Forschungsprojekten gehören bisher die z.T. noch in Überarbeitung befindlichen Dokumentationen und Ressourcen der Projekte „Grenzgang“ und „To Perform“. Bei beiden waren auch die Musikhochschulen FHNW involviert, wenn auch auf sehr unterschiedliche Weise: Während bei

15 Da die Metadaten frei recherchierbar sind, werden die sich wandelnden Themenschwerpunkte innerhalb der Abschlussarbeiten der Institute erkennbar.
„Grenzgang“ die „künstlerische Untersuchung zur Wahrnehmung und Vermittlung von Raum im trinationalen Grenzgebiet" in der Region Basel (CH/D/F) im Zentrum stand und viele Materialien, künstlerische Interventionen etc. im Rahmen von Spaziergängen realisiert, gesammelt und teilweise für die Kunstvermittlung (z. B. in Schulen) aufbereitet wurden, untersuchte „to perform“ Aufführungspraktiken der Neuen Musik. Hierzu wurden die vier Komponisten Irvine Arditti, Peter Eötvös, Peter Maxwell Davies und Vinko Globokar bei der Vorbereitung zu Aufführungen ihrer Werke (Konzerte) videografisch begleitet, beobachtet und interviewt. Die Beobachtung des Übens und Probens verdeutlicht die heute kaum noch über Notationen abbildbare Komplexität musikalischer Aufführungspraxis, die durch derartige Ressourcen für die Nachwelt besser nachvollziehbar gehalten werden soll.

Unter den bereits digital erschlossenen Sammlungen der Lehrmittel \& Kooperationen seien folgende erwähnt:

- Die Videosammlung des Instituts Kunst wurde von dem Schweizer Künstler, Kurator und Kunsttheoretiker René Pulfer in seiner langjährigen Tätigkeit als Leiter des Instituts Kunst zusammengetragen. Von den ca. 1100 Medien wurden bisher bereits knapp 600 online erschlossen, sodass sie abhängig vom Rechtes- 
tatus der Nutzer direkt im Browser gesichtet werden können.

- Der Online Zugang zur „Digitalen See“ geht auf das Umfeld der ehemaligen Dozentin und Schlüsselfigur der Basler Performancekunst Muda Mathis zurück, die in langjähriger Arbeit mehrere Stationen der „Performance Chronik Basel“ aufgearbeitet hat. Im Sommer 2017 wurde für den Abschnitt 1996-2006 der online Zugang zu 178 Einträgen eingerichtet, ${ }^{16} \mathrm{zu}$ dem in Kürze ein Teil der Sammlung Basler Performanceraum Kaskadenkondensator (Kasko) hinzukommt, das ca. 160 Dokumentationen aus den Jahren 1998/99-2008 umfassen wird. Diese Ressourcen sind Bestandteil der „Digitalen See“, die verschiedenste Performanceaktivitäten dokumentiert.

- Die Online-Sammlung Videokunst.ch wurde hingegen nur metadatentechnisch eingebunden und weist die Nutzer auf deren Webseite. Sie gehört zu jenen Kooperationsprojekten, die als Schaufenster auf externe Sammlungen den Rechercheweg verkürzen und die Kenntnis der Plattform fördern.

Mit Blick auf Schenkungen \& Capta, die teilweise als externes Konvolut eingebunden wurden, seien erwähnt:

- Die Sammlung interaktiver CD-ROMs und DVDs von Matthias Zehnder. Sie umfasst ca. 3300 Datenträger aus der Zeit zwischen 1994 und 2001, zu denen in den meisten Fällen die Begleit-Bocklets und Originalverpackung vorhanden sind. Die Schenkung vermittelt ein Überblick über unterschiedlichste Themenfelder aus der Blütezeit der interaktiven CD-ROM (und DVD), ${ }^{17}$ was sowohl grafisch, als auch mit Blick auf das Interaktionsdesign oder auch die kultur- und mediengeschichtlichen Implikationen spannend ist.

- Die Schenkung des Printarchivs des Birkhäuser-Verlags gelangte nach der Auflösung der Basler Büroräume an die HGK. Während je ein Sichtungsexemplar in den kommenden Monaten via NEBIS erfasst und zur Verfügung gestellt wird, erfolgt die Dokumentation der Archivbestandteile (Mehrfachexemplare, Überarbeitungsexemplare etc.) nur über eine Zotero-Liste,

16 Mediathek HGK (2017).

17 Da international vernetzte Emulationsprojekte wie BWFLA (Emulation as a Service, http://bw-fla.uni-freiburg.de/) und ihre Nachfolger heute bereits historische Rechnersuiten über die gängigsten Standardsysteme anbieten, in welche die Datenimages der CD-ROMs und DVDs gemauntet werden können, wird eine Ripp-on-Demand-Zugänglichkeit geplant, bei der erfolgreich gesicherte Datenträger dann auch ausgeliehen werden können. Während die Metadaten bereits recherchierbar sind, läuft die Planungsphase zur operativen Umsetzung noch. die in einem Spezialbereich des integrierten Katalogs der Forschung zur Verfügung gestellt wird.

- Die Dokumentation der „Videowochen Wenkenpark“ umfasst 40 Videoarbeiten und zwei Fotodokumente der drei heute legendär gewordenen Videowochen 1984/1986/1988. An den in Riehen veranstalteten Videowochen haben heute international gefeierte Künstler wie Dara Birnbaum, Dan Graham, Nan Hoover, Bruce Nauman, Terry Fox und andere teilgenommen und z. T. Konzepte (vgl. Gary Hill) entwickelt, die später auch als eigenständiges Werk in Umlauf kamen. Dank René Pulfer und Manfred Manz ist die Affiliierung des „Wenkenpark“-Projektes an der HGK nie abgerissen, sodass das durch den Verein zur Erhaltung des audiovisuellen Kulturgutes der Schweiz (Memoriav) geförderte Projekt nun in der Mediathek zugänglich gemacht werden kann.

- Die Sammlung der Capta ist noch im Aufbau begriffen und versammelt hilfreiche Online-Dokumente und Portale (z. B. zu Datenbanken), wie sie viele Institutionen im Kontext ihrer Link- oder Referenzlisten vorhalten. Das Problem hierbei ist, dass diese Links dann, wenn sie gebraucht werden, also während der Recherche, nicht auffindbar sind, weil sie ja „nur“ eine Liste, aber kein Metadateneintrag im Katalogsystem sind. Mithilfe der Zotero-Workflows lassen sich solche Ressourcen im integrierten Katalog erschließen und ausweisen.

Zudem hat die Mediathek begonnen, mit aktuellen Methoden an die existierenden Sammeltraditionen der HGK anzuknüpfen.

- Exemplarisch für diese neuartigen Akquisitionswege sei die Zusammenarbeit mit dem Basler Film- und Videofestival „, Summe 2017“ erwähnt, dem wir einen vollständig digitalen Einreichungsprozess ermöglicht haben. Während sich die Unterstützung inhaltlich aufgrund der lokalen Vernetzung sowie persönlicher Bezüge auf der Ebene der Studierenden, des Mittelbaus, der Dozierenden und diverser Alumni anbot, eröffnete die Bereitstellung einer Plattform den teilnehmenden Künstlern administrativ die Möglichkeit, der Mediathek direkt bei der Einreichung definierte Nachnutzungsrechte zu übertragen. ${ }^{18}$

18 Von den 198 Einreichungen dürfen 13 Arbeiten an einer definierten Sichtungsstation der Mediathek, 16 in der Mediathek insgesamt 15 Arbeiten in der HGK FHNW, 67 im Schweizer Hochschulnetz und 62 frei im Internet gezeigt werden. 
Alle Datensätze werden programmiertechnisch über ihre jeweilige Kennung (ISIL, Bib.-Kennung, Bestands-ID etc.) adressiert, sodass nicht nur die Teilbestände, sondern auch die virtuellen Sammlungen flexibel gruppiert, erweitert oder reduziert werden können. Nutzerseitig gleicht das Vorgehen einer Facettierung, die via Mausklick am rechten Rand erfolgt. Da sich die Suchergebnisse sammlungsübergreifend mischen können, werden bei jedem Datensatz bereits in der Listenvorschau die jeweiligen Datenlieferanten referenziert und zwar bei der ID, vor der Nennung der Systemnummer/Sammlungs-ID. ${ }^{19}$ Die Sichtbarkeit der Datenprovider kann auch zur Beurteilung der Qualität, der Relevanz, als Kontextwissen der Quellen sowie zur weiteren Recherche hinzugezogen werden. Während die Heterogenität der Dateiformate durch die Darstellungskonvention (s.u.) abgefedert wird, tragen harmonisierende Workflows bei der Erfassung der Inhalte heute dazu bei, dass die Metadatenqualität auf ein in etwa einheitlich gutes Niveau gehoben wird.

\subsection{Workflows und Prozesse}

Der integrierte Katalog ist aber nicht nur eine Rechercheinfrastruktur, sondern er erfüllt hochschulintern die Funktion, die dokumentarischen Workflows und Austauschprozesse zu strukturieren. Das erleichtert nicht nur die Zugänglichkeit der Materialien, sondern regelt (rechtetechnisch) auch die (Nach-)Nutzung und entlastet mithin die Institute. Eine Herausforderung und zugleich große Chance besteht daher in der Einbindung der Hochschulangehörigen und Institute. Als Experten ihrer eigenen Disziplinen haben diese Personen nur überschaubare Kapazitäten zur (Unterstützung der) systematischen Erfassung von Metadaten oder der Beschreibung ihres Vorgehens. Da sie aber über spezifische Fachkenntnisse und einen Instinkt für aktuelle Themen, Materialien, Belange etc. verfügen, sind einfache, plattformübergreifende Werkzeuge und simple Workflows gefordert, die eine rasche, verteilte Erfassung von Inhalten ermöglichen, anpassungsfähig sind und auch mal Externe einbeziehen können. ${ }^{20}$ Dabei unterscheiden wir:

19 Im obigen Beispiel (Abb. 1) stammen die beiden aufgeschlagenen Datensätze aus der swissbib, der Link für die Standortangaben (Männchen) stammt aus dem Inventursystem der Mediathek.

20 Plattformübergreifend meint primär Macs und PCs, aber auch Linux-Systeme. Die Einbeziehung Externer erfordert freie, Open-Access-Software und eine kommunikative Plattform, die damit umgehen kann, dass nicht alle Nutzer Hochschulangehörige sind. Beides ist auch für Studierende relevant, da sie nach dem Studium i.d.R. auf
- Eine Zotero-Suite zur Erfassung aktueller und Übernahme von älteren Beständen: Ein wichtiges Werkzeug im Pre-Ingest-Prozess ist die Bibliografie-Software Zotero. ${ }^{21}$ Unsere Zotero-Suite (inkl. Workflows) kommt bei Materialserien, im Kontext der Retro-Katalogisierung, bei Buch- oder Medienbeständen, die nicht über den ALEPH verwaltet werden (z. B. VorratsDubletten, Spezialsammlungen vgl. Birkhäuser etc.), bei der Lehrmittelverwaltung (Reader, Handapparate, Materialserien) sowie bei der Pflege der Instituts- und Hochschulbibliografie(n) zum Einsatz. Mit Blick auf die Datenerfassung (Ingest-Kontext) besteht der Vorteil darin, dass die Metadaten in Tabellen vorbereitet werden können, bevor sie in spezifische Zotero-Sammlungen importiert werden. Die Editoren können im Anschluss an den Import und die Bereitstellung in der Zotero-App Korrekturen vornehmen, die Medien neu gruppieren oder Ressourcen (Dateien /Dokumente) anhängen. Die Aufbereitung der Medien fürs Web erfolgt über ein Transkodierungscluster. ${ }^{22}$ Der Prozess geht schnell, ist selbsterklärend und ist für die Editoren auch ohne spezifische Vorkenntnisse machbar. Aus administrativer Sicht konnten dank Standardisierung Templates für die Detailseiten der unterschiedlichen Medientypen entwickelt werden, welche die Wiedergabe und Layouts häufig wiederkehrender Metadaten-Material-Kombinationen im integrierten Katalog weitgehend automatisierbar machen: Vom Zotero aus werden die Metadaten, Inhalte und Ressourcen über eine standardisierte Erfassungskaskade analysiert und in den Volltext-Index importiert. Anschließend erfolgten die Überprüfung des Layouts und die Ausspielung in den integrierten Katalog. Künftig können auch weitere Subsysteme wie etwa Webseiten, Blogs etc. mit Inhalten bedient werden. ${ }^{23}$

- Formularbasierte Erfassung: Die Erfassung von Einzelressourcen durch eine heterogene Zielgruppe, die sowohl zeitlich, als auch materialtechnisch und/oder

viele lizensierte Produkte verzichten müssen, wenn sie ihre Hochschulaffiliierung verlieren.

21 Rosenzweig (2017).

22 Aus Textdateien (Word, PDF etc.) werden die Rohtexte extrahiert und im Volltextindex abgelegt. Mediendateien werden mithilfe von Tools wie imageMagick (Bild), Spatial Audio Coding SAC (Audio sowie für Sonogramme ein selbst in C++ entwickeltes Programm) oder GPSBabel (Positionsdaten) in ein webtaugliches Format überführt. Die Videotranscodierung erfolgt aus Kostengründen in der Claud (ZENCODER).

23 Der Grad der Automatisierung hängt von der Bereitschaft der Zielgruppen ab, sich an Standards, Gestaltungsregeln und/oder Systemkompatibilitäten zu halten. 
personell unüberschaubar ist, erfolgt derzeit durch Formulare. Exemplarisch seien die Erfassung der Diplombeiträge für die DiplomHGK-Webseite 2017 oder auch die Kooperation mit der „, Summe 2017“ genannt. Diese Inhalte werden zwar direkt vom Formular aus in eine MySQL-Datenbank geschrieben, aber dann erfolgt zunächst ein Export ins Zotero, bevor die ggf. korrigierten oder angereicherten Inhalte in den Volltext-Index importiert werden (s. o.). Grund dafür ist die häufig gebotene Überprüfung/Korrektur einzelner Angaben. Der Einsatz dieser Upload-Formulare spart nicht nur Ressourcen, ist aus rechtlicher Sicht vorteilhaft und reduziert Fehlerquellen, sondern erleichtert auch die Akzeptanz für digitale Workflows.

Es ist gut möglich, dass die Erfassungsworkflows künftig noch ergänzt werden, wenn das institutionelle Repository zur Verfügung steht. Ob die skizzierten, niederschwelligen Erfassungsroutinen grundsätzlich abgelöst werden, wird sich zeigen.

\section{Katalogkompetenz}

Katalogkompetenz meint ein beiderseitiges Streben, das nicht nur von den Nutzern spezifische Kenntnisse und Übung erfordert, wenn sie zielführend recherchieren und sich im Katalog zurechtfinden möchten: „,information literacy is a 'learning issue not a library issue"“, wie David Walczak et al. mit Blick auf die längerfristige Entwicklung dieser Kompetenz erläutern. ${ }^{24}$ Katalogkompetenz ist auch mediatheksseitig eine kontinuierliche Aufgabe und ein Ziel, denn der Katalog ist die kommunikative Schnittstelle, welche Austauschprozesse ermöglicht. An der Katalogoberfläche treffen mithin Aspekte der Nutzerfreundlichkeit (Usability) und der inhaltlichen Aufbereitung der Daten (ContentDesign) auf Prinzipien der Informationskompetenz und der Nutzeraktivierung. Letzte lassen sich z.B. mithilfe der ABCD-Methode von Dominique Turnbow und Annie Zeidman-Karpinski umreißen, die zwar der Zielentwicklung bei der Vermittlung von Informationskompetenz dienen soll, aber auch andere Aspekte des Qualitätsmanagements in

24 Walczak et al. (2010) 194. Während der geforderte Perspektivwechsel, der die bibliothekarische (Innen-)Sicht auf Kataloge und Datenbanken verlässt, hilfreich ist, um die Bedürfnisse der Nutzer an den Anfang zu stellen, wird die Kontinuität in der Beschäftigung mit wissenschaftlichen Fragen in der HGK nicht nur über den Klassenraum, also wiederkehrende Lehreinheiten im Curriculum, sondern auch über die Mediathek als dauerhafte Anlaufstelle und Lernraum gewährleistet.
Bibliotheken betreffen kann. Sie thematisieren die Nutzer (A = audience), ihr Verhalten ( $\mathrm{B}=$ behavior), ihre Befindlichkeit und die Bedingungen, untern denen sie arbeiten/recherchieren $(\mathrm{C}=$ condition) sowie ihren Lernerfolg $(\mathrm{D}=$ degree). ${ }^{25}$ Besonders wichtig sind in der Mediathek die Rahmenbedingungen (C), die sowohl den physischen Lernraum und sein Ermöglichungspotential, als auch das Nutzerinterface des Kataloges betreffen.

\subsection{Usability \& Content-Design}

Bei der Gestaltung der Nutzeroberfläche des integrierten Katalogs waren uns daher nicht nur die bibliothekarischen Standpunkte wichtig, sondern auch und primär eine nutzerfreundliche Aufbereitung der Informationen. Die Erscheinungsweise des integrierten Katalogs wird im Folgenden vor dem Hintergrund der Principles for Usable Design betrachtet, die von der User Experience Professionals ${ }^{\star}$ Association zwischen 2002 und 2015 entwickelt wurden. ${ }^{26}$ Sie heben sieben Grundprinzipien hervor, welche die Nützlichkeit (usefulness) der Webseite und ihrer Inhalte, die Konsistenz (consistency) derselben, ihre Einfachheit (simplicity), die kommunikative Struktur (communication), die Fehlervermeidung und die Handhabung (error prevention and handling), die Effizienz (efficiency), die Arbeitsentlastung (workload reduction) sowie die Benutzerfreundlichkeit (usability judgement) betreffen. Für die vorliegende Erläuterung haben wir die Reihenfolge gemäß unseren Prioritäten angepasst:

- Simplicity: Einfachheit ist die wichtigste Maxime des integrierten Katalogs. So führt auch eine leere Suche zu Ergebnissen, die ohne weiteres Zutun schlicht in 12 Zeilen untereinander aufgelistet werden. Schlicht meint, dass mit einem Minimum an gestalterischen Elementen gearbeitet wurde: schwarze Schrift und Ikons vor weißem Hintergrund, gegliedert durch anthrazitfarbene Linien, nur rechts Kästen zur Auswahl der Kataloge und Sammlungen sowie der Facetten und schließlich einem anthrazitfarbenen Balken ganz recht, der erklärt, wo man sich befindet (Ebene $1=$ Suche, Ebene 2 = Details). Ein Standardicon klassifiziert den Medientyp und strukturiert die Angaben, indem es zwischen dem/den Autor/en (links) und dem Titel (rechts) eine visuelle Zäsur (1/3 zu 2/3) setzt. ${ }^{27} \mathrm{Um}$

25 Turnbow und Zeidman-Karpinski (2016) $146 \mathrm{f}$.

26 Vgl. hierzu User Experience Professionals Association (20052012).

27 Je nach Ressourcentyp, Medienformat und teilweise auch abhängig vom Sammlungszusammenhang werden Ionicons, http://ioni 
das Scrollen zu vermeiden, öffnet sich die Listenansicht erst beim einmaligen Anklicken (VorschauAkkordeon), um dann ausführlichere Titelinformationen zu liefern. Bei elektronischen Ressourcen führt ein Link direkt auf die Daten. Bei analogen Medien enthält eine graue Figur hinter der Signatur einen Link zum dynamischen full-3D-Modell, welche in einem neuen Fenster den Standort im Raumzusammenhang aufzeigt. Die Detailseiten folgen trotz der Heterogenität der Inhalte und Formate einem gleichbleibenden Raster: oben links findet sich der Kernmetadatensatz, in der Mitte sind ein Vorschaubild oder die digitale Ressource (Text, Bild, Video- oder Audiofile [repräsentiert mittels Sonogramm], blätterbare PDFs, GPX-Track etc.) sowie bei NEBIS-Medien der Mediathek die 3DStandortanzeige zu sehen. Rechts führen Suchbegriffe zu einer erneuten Suche. Im unter Seitenbereich wird bei kuratierten Objekten und Sammlungen der Kontext angezeigt, also weitere zugehörige Medien. Bei Büchern des NEBIS-Bestandes sind dies die übrigen Medien der gleichen Regalbox (thematische Klassifikation), bei studentischen Arbeiten die Beiträge der anderen Seminarteilnehmer/Absolventen (Jahrgang, Kurs, Jahr), bei Forschungsclustern die übrigen Inhalte der Sektion und bei OA-Journals die anderen Aufsätze des gleichen Bandes (issues).

- Usefulness: Im Hinblick auf die Nützlichkeit unterscheiden die Autoren der Principles zwischen dem Wert und der Relevanz der Informationen einer Webseite. Auch wenn leicht der Eindruck entstehen kann, dass der Nutzen eines Medienkatalogs allein schon aufgrund der Fülle an Materialien selbsterklärend ist, sollte dies nicht zum Selbstzweck verkommen. Die angezeigten Inhalte sollten zielführen sein (relevance), was sich im integrierten Katalog z. B. in der Listenansicht zeigt: die Nutzer sind mit zwei Klicks (Schuhbegriff, Anklicken der Ressource $=$ Listenvorschau am Ziel, da sie ihr Medium mit Signatur und Standort im Raum finden oder direkt online angezeigt bekommen.

- Consistency: Konsistenz beschreibt das Wechselspiel zwischen der Einhaltung von Standards und dem alltäglichen Erfahrungsschatz. Mit Blick auf die Konsistenz wird im integrierten Katalog zwischen der Menschen- und der Maschinenlesbarkeit unterschieden, also der sichtbaren Oberfläche der Webseite und dem HTML5-Code. Während die Metadateneinträge ästhe-

cons.com/ bzw. Bilder von Fontawesome, http://fontawesome.io/i cons/ verwendet, die als allgemeinverständlich betrachtet werden dürfen (visibility \& self-evidency). tisch durch variierende Layouts voneinander abgegrenzt werden, sind die Informationen auf Codeebene semantisch klassifiziert, sodass die Seiten maschinenlesbar sind. Mit Blick auf die Qualitätssteigerung durch Standards sei erwähnt, dass eine Integration von metagrid.ch geplant ist, welche z.B. Personenansetzungen unseres Eigenbestands auf die GND-Ansetzungen für Personen sowie die IDs im SIKArt-Lexikon mappt. ${ }^{28}$

- Communication: Kommunikative Aspekte betreffen gemäß den Principles die Reaktionskultur der Seite (Feedback), die Strukturierung der Informationen und die Gruppierung (Sequenzierung) der Inhalte oder Aktionen. Mit Blick auf das Feedback wäre im integrierten Katalog das Prinzip „ein Klick, ein Treffer“ hervorzuheben, das e-Ressourcen direkt aus der Listenvorschau verlinkt. Mit Blick auf die semantische Strukturierung der Inhalte kann das digitale Kuratieren der Daten angeführt werden, das inhaltliche Zusammenhänge abbildet.

- Efficiency: Bezüglich der Effizienz wird in den Principles z. B. eine Vorschlagsystematik empfohlen, die den Nutzern ein „continuous advancement in knowledge and skill“ ermöglicht. ${ }^{29}$ Abkürzungen (im System) und das selbstbestimmte Fortbewegen, das die Prinzipien weiter fordern, bieten Detailseiten aber auch das sog. „Kistentool“, welches neben der integrierten Lösung im Katalog auch als separate Seite zur Verfügung steht und als Antwort auf die Eingabe von Signatur oder Strichcode (Exemplarsatzkennung) den Standort ausgibt. Diese Verknüpfung von realem und virtuellem Raum ist in der Mediathek aufgrund der komplexen Raum- und Regalstruktur wichtig. ${ }^{30}$

- Workload Reduction: Zur Reduktion von Komplexität empfehlen die Principles einfache Verfahrensweisen und Interfaces, welche die mentale Leistung zur Orientierung minimieren. Zudem soll Redundanz vermieden werden. Ein Vergleich des integrierten Katalogs mit üblichen Bibliothekskatalogen oder Recherchesystemen verdeutlicht, dass diese Aspekte angegangen wurden. Beschreibungsfelder, welche Metadaten

28 Das Künstlerlexikon des Schweizerischen Instituts für Kunstwissenschaft SIK ISEA (Zürich) findet sich unter http://www.sikart.ch/ku enstlerinnen.aspx.

29 User Experience Professionals' Association (2005-2012).

30 Statt klassischer Regalreihen präsentiert die Mediathek ihre analogen Medien in sowohl horizontal als auch vertikal geschwundenen Bögen, die aus im Versatz gestapelten Regalboxen (statt Tablaren) bestehen. Die Kombination dieser Dynamik mit 7 historisch gewachsenen Signaturensystematiken hat dazu geführt, dass wir 2016 eine umfassende Inventur durchgeführt haben, bei der die Metadaten der RF-IDs konkreten Standorten zugewiesen wurden (Inventursystem). 
erklären, werden nur dort angeführt, wo dies wirklich nötig ist, wie beispielsweise die Klassifikation der Betreuer von Abschlussarbeiten, die Benennung von Künstlern, welche z.B. die Kamera bei der Dokumentation betätigt haben (und mithin nicht die Autoren des Werkes sind) etc. Zur Orientierung tragen auch die Auswahlkreuze der angewählten Sammlungen (Rechercheebene) sowie die Benennung des Sammlungskontextes auf den Detailseiten (oben) bei.

- Error Prevention and Handling: Bezüglich der Fehlertoleranz werden das Rückgängigmachen von Fehlentscheidungen (Backtaste), das Zurücksetzen der Eingabemasken sowie Notausgänge (undo and redo) angeführt. Bei uns kann man im Katalog nichts „kaputt“ machen. Außer der Zurück-Option (Backtaste) wurde versucht, die Erwartungshaltung der Nutzer in der Verlinkungsstruktur zu berücksichtigen. So wird z. B. zwischen einer einschränkenden Suche des Teilergebnisses (Facettierung) auf der Suchseite und einer neuen Suche beim Anklicken von Keywords oder Autoren auf den Detailseiten unterschieden. Das Stöbern innerhalb der Einträge einer Detailseite baut das Suchergebnis hingegen nicht erneut auf.

- Usability Judgment: Aus dem Blickwinkel klassischer Bibliothekskataloge haben wir mit einigen Konventionen gebrochen. Wir haben nicht nur die Ressourcen direkte im Suchergebnis verlinkt, sondern wir haben auch auf eine explizite Detailsuche verzichtet. Wer möchte, kann mit eine standardisierten Syntax eine Spezialsuche lancieren. ${ }^{31}$ Mit Blick auf die nichtbibliothekarischen Bestände wurde erst einmal auf RDA und MARC21 verzichtet. Da alle Metadaten im SOLR-Index aber als Rohdatensatz gespeichert sind, kann trotz der Reduktion von Komplexität bei der Wiedergabe (Detailseiten) auf die ursprüngliche Erfassungstiefe (und -konvention) zurückgegriffen werden. Die Darstellungstiefe der Metadaten orientiert sich an üblichen Vorgaben zur Erstellung von Literaturverzeichnissen bei wissenschaftlichen Arbeiten. ${ }^{32}$ Bei eigenen Sammlungsbeständen werden die Detailseiten gemäß den jeweils spezifischen Anforderungen gestaltet (Sonderlösungen inklusive).

31 Die Suche nach Personen lautet dann „author:Lastname,firstname“, jene nach dem Titel „title:xyz“.

32 Alle weiteren Informationen sind bei Buchmedien und Zeitschriften über den NEBIS (via Klick auf die Signatur der Detailseite) bzw. die EZB ersichtlich. Diese Referenz ist auch wichtig, weil wir in unserem Katalog die Fernleihinformationen derzeit nicht anzeigen. Die Bestellabwicklung erfolgt via NEBIS-Interface und das soll für die Nutzer auch so ersichtlich sein.
Das Content-Design betrifft beim integrierten Katalog neben der Oberflächengestaltung der Detailseiten vor allem die eher unsichtbare Arbeit des digitalen Kuratierens der Inhalte, also die Frage, wann welche Ergebnisse angezeigt werden. Auch wenn das bisherige Information Retrieval auf eher einfachen Heuristiken wie den Kernmetadaten, der manuellen Gewichtung der Medientypen sowie der Sammlungszugehörigkeit basiert, sind für die Zukunft Kuratierungsoptionen geplant, die eine fachspezifische Aufbereitung der Inhalte erleichtern und die Hochschulangehörigen noch expliziter einbeziehen. So sollen individualisierbare und/oder domänenspezifische Sichtweisen auf die Inhalte entstehen, welche die Institutsinteressen besser abbilden.

\subsection{Werkzeuge}

Die Entwicklung digitaler Werkzeuge erfolgte in der Mediathek von Anfang an auf der Basis spezifischer Anforderungen: Um die zunächst unübersichtliche Raum- und Bestandssituation in den Griff zu bekommen, wurde ein RF-ID-basiertes Inventursystem entwickelt, das nicht nur die Informationen der RF-IDs erfasst, sondern den damit verknüpften Medien auch einen (physischen) Standort zuweist. Neben einem Backup für die „korrekte“ Information der RF-IDs wurde für diesen Datenbestand das sog. „Kistentool“ entwickelt, welches eine Brücke vom realen Raum, seinen Denktraditionen und dem Katalog mit seinen verschiedenen Strukturierungsvorlieben schlägt und die eigenständige Orientierung ermöglicht.

Als weiterer Mangel wurde das Handling von OpenAccess-Quellen im Primo-Interface (Exlibris) des NEBIS empfunden. Es scheint OA nicht zu fördern, sondern zu diskriminieren, weil alle explizit als OA gekennzeichneten Quellen in dem Reiter ,articles and more“ ausgewiesen werden. Um die Hochschulressourcen besser dokumentieren und erschließen zu können, wurde der integrierte Katalog entwickelt, der eng mit dem Inventursystem verknüpft ist.

Weil die RF-ID-basierte Diebstahlsicherung der sog. Gates an den Eingängen der Mediathek Unregelmäßigkeiten zwar mittels Alarm angezeigte, die Überprüfung der konkreten Störfälle in der Cloud auf uns aber völlig unzureichend wirkte, haben wir eine eigene Gatesoftware entwickelt, die über die gleiche Plattform wie der integrierte Katalog in Echtzeit einsehbar ist. Im Unterschied zum kommerziellen Anbieter werden darin alle verfügbaren Informationen direkt ausgewiesen (inkl. Nutzungsstatistik), es wird geprüft, ob es sich um eigene oder Fremdmedien handelt (ISIL) und ob unsere Chips manipuliert wur- 

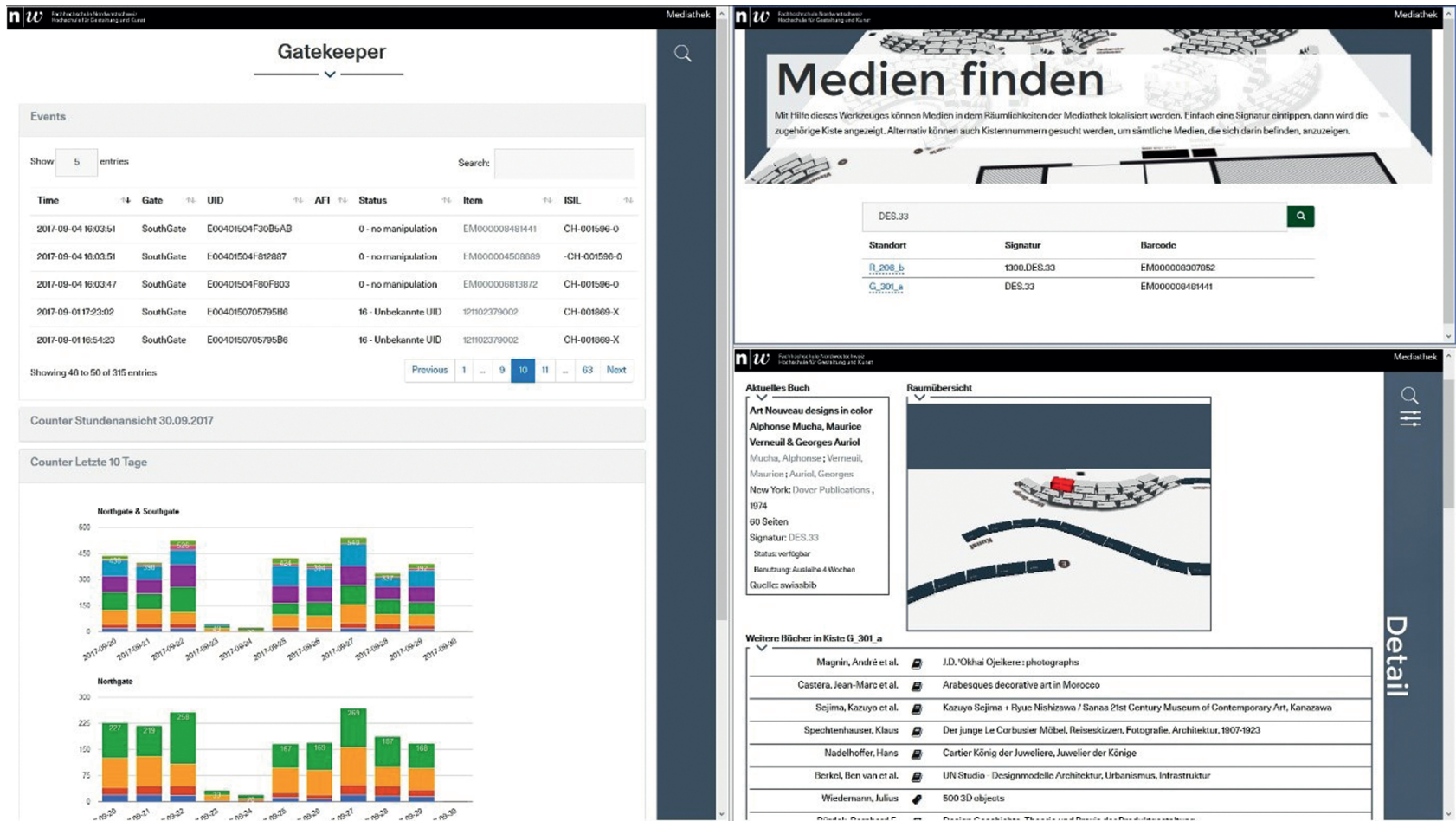

Abb. 4: Mediathekswerkzeuge mit Kataloganschluss: Links: Der Gatekeeper (links) zeigt oben den Zeitpunkt des Alarms, das betroffene Gate, sowie UID, AFI-Wert und Manipulationsstatus der betroffenen RD-ID sowie den Strichcode und die ISIL des zugehörigen Mediums. Rechts oben ist das Kistentool mit Standort, Signatur und Strichcode sowie darunter eine Detailseite zu sehen, die sich öffnet, wenn im Gatekeeper der Strichcode angeklickt wird.

den. Der Exemplardatensatz (Strichcode) ist in diesem Tool direkt in den NEBIS verlinkt, was die Identifizierung auch rückwirkend erleichtert. Der Gatekeeper verschlankt nicht nur die Kontrollroutinen, sondern erhöht künftig das Sicherheitslevel: Während das vorherige Systeme lediglich die AFI auf dem Tag überprüft, welche den Ausleihstatus anzeigt, findet bei uns sowohl ein Abgleich mit der Inventurdatenbank (Konsistenzcheck), als auch künftig eine Überprüfung des Ausleihstatus im Katalog (Sip2 Schnittstelle) statt.

Als jüngstes Toolset könnten die Zotero-Suite, die Erfassungsformulare für den Ingest sowie ein Video-Player erwähnt werden, der aus einem vorselektierten Videopool zufällig eine manuell einstellbare Anzahl von Treffern auswählt, diese im Browser bildschirmfüllend darstellt und dann vollautomatisch ein Medium nach dem anderen abspielt.

\section{Fazit und Ausblick}

Die Integration von Bibliotheks-, Forschungs- und externen Fachdatenquellen in einem zentralen Portal repräsentiert für uns jenen fließenden künstlerisch-gestalterischen
Wissenskreislauf, den wir in Anlehnung an klassische Forschungsdatenzyklen als „Creative Data Cycle“ bezeichnen. Der „Creative Data Cycle“ benennt die Schnittstellen der Lehre und Forschung an der HGK mit Daten sowie digitalen Werkzeugen, Infrastrukturen und Prozessen. ${ }^{33}$ Der integrierte Katalog ist also nicht nur eine wichtige Rechercheinfrastruktur, die künftig auch in die (neue) CampusWebseite eingebunden sein wird, sondern in ihm kreuzen sich unterschiedliche Datenströme. Austauschprozesse und Kommunikation werden beim Einbringen neuer (oder alter) Daten besonders greifbar, da hier häufig nicht nur Einzeldatensätze erfasst werden, sondern ganze Datensets, deren Kontext es zu erhalten und überliefern gilt. Es ist evident, dass sich diese Systeme an die Bedürfnisse und Konvention der Nutzer anpassen, wo immer dies möglich ist.

Dabei bleibt der integrierte Katalog, was er ist, nämlich ein Katalog. Er ist weder ein Datenspeicher noch ein Archiv. Er verweist nur auf jene Ressourcen, die nach dem

33 Die Beschreibung der konzeptuellen Verankerung des „Creative Data Cycles“ der Mediathek HGK ist im Konferenzband zum Frankfurter Bibliothekartag 2017 erscheinen (https://doi.org/10.5282/obib/ 2017H4S162-175). 
Open Archival Information System (OAIS) als Dissemination Information Package (DIP) bezeichnet werden. ${ }^{34}$ Er verkürzt die Zugriffswege im Frontend, indem er die Anzahl der Klicks minimiert, und strafft die Wertschöpfungskette im Backend, da er - wo immer möglich - auf digitale Zwischenhändler verzichtet. ${ }^{35}$ Das technologische Rückgrat des integrierten Katalogs ist ein SOLR-Volltextindex, der jederzeit neu aufgebaut werden kann, weil diese Aktualisierung lediglich zeitintensiv aber nicht datentechnisch kritisch ist. Die hauseigenen Daten und Metadaten lagern derzeit noch in vernetzten Zwischensystemen (PostgreSQL-/MySQL-Datenbank), von denen aus die Daten in den Volltextindex eingebracht werden. Umfangreiche Datenquellen wie jene der swissbib werden nicht zwischengespeichert, sondern direkt in den Index integriert. Künftig wird das Datenmanagement über ein Institutional Repository geregelt, das auf einer DSpace-Instanz basieren wird, da so die FAIR Data Principles eingehalten werden können und eine euroCRIS-Erweiterung für Forschungsdaten möglich wird. ${ }^{36}$ Die Implementierung erfolgt, wie bisher, durch das Center for Digital Matter (Campus-IT). Als Open-Source-Software implementiert, findet sich der Code auf Github, ${ }^{37}$ wo auch Neuerungen ersichtlich sind.

\section{Literaturverzeichnis}

Bennett, Hannah (2006): Bringing the Studio into the Library. Addressing the Research Needs of Studio Art and Architecture Students. In: Art Documentation: Journal of the Art Libraries Society of North America, 25 (1), 38-42. https://doi.org/10.1086/ adx.25.1.27949400.

euroCRIS (Current Research Information Systems) (Hrsg.) (2017): Welcome to the euroCRIS DSpace CRIS digital repository. Verfügbar unter http://dspacecris.eurocris.org/.

FORCE11 (The Future of Research Communication and e-Scholarship) (Hrsg.) (2011-2017): The FAIR Data Principles. Verfügbar unter ht tps://www.force11.org/group/fairgroup/fairprinciples.

Hipler, Günter (Hrsg.) (2017): Open Science Track @OpenData.ch/ 2017. „Data Processing als Kern/Basis einer offenen Schweizer Metadatenplattform“. Verfügbar unter https://opendata.ch/wor dpress/files/2017/01/opendata.luzern.2017.hipler.pdf.

International Organization for Standardization (Hrsg.) (2012): Open archival information system (OAIS). Reference model. ISO 14721:2012 (CCSDSS 650.0-P-1.1). Space data and information transfer systems. Verfügbar unter https://www.iso.org/contactiso.html.

Kitchin, Rob; Dodge, Martin (2011): Code/space. Software and everyday life (Software studies). Cambridge Mass.: MIT Press.

Lauwaert, Maaike; van Westrenen, Francien (Hrsg.) (2017): Facing value. Radical perspectives from the arts. Amsterdam: Valiz; Stroom.

Mediathek HGK (Hrsg.) (2017): Online Zugang zur Performance Chronik Basel. Verfügbar unter https://mediathek.hgk.fhnw.ch/ pcb.php.

MIZ (2017): nanoo.tv, hrsg. v. Medien- und Informationszentrum (Zürcher Hochschule der Künste). Verfügbar unter https://www.zhd k.ch/miz-nanootv.

Rosenzweig, Roy (2017): About Zotero, hrsg. v. Center for History and New Media George Mason University. https://www.zotero.org/ about/.

Turnbow, Dominique; Zeidman-Karpinski, Annie (2016): Don't Use a Hammer When You Need a Screwdriver. How to Use the Right Tools to Create Assessment That Matters. In: Communications in Information Literacy, 10 (2), 143-162. https://doi.org/10.7548/ cil.v10i2.438.

User Experience Professionals Association (2005-2012): Usability Body of Knowledge. Principles for Usable Design. Verfügbar unter http://www.usabilitybok.org/principles-for-usable-de sign.

Walczak, David A.; Sammet, Diane L.; Reuter, Monika E. (2010): A Program for Introducing Information Literacy to Commercial Art and Design Students. In: Communications in Information Literacy, 3(2), 193-203. https://doi.org/10.7548/cil.v3i2.81.

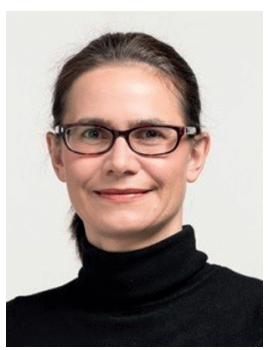

Tabea Lurk

Hochschule für Gestaltung und Kunst FHNW Mediathek

Freilager-Platz 1

CH-4002 Basel

Schweiz

tabea.lurk@fhnw.ch

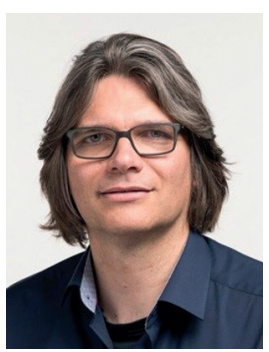

Jürgen Enge

Hochschule für Gestaltung und Kunst FHNW Center for Digital Matter

Freilager-Platz 1

CH-4002 Basel

Schweiz juergen.enge@fhnw.ch

34 International Organization for Standardization (2012).

35 So können Verlagsinhalte (vgl. OA-Inhalte) direkt ins eigene System eingebunden und aufbereitet (z. B. geclustert) werden.

$36 \mathrm{Zu}$ den FAIR-Principles vgl. FORCE11 (2017), zu EuroCriss vgl. EuroCRIS (2017).

37 Die Github-Dokumentation findet sich unter https://github.com/j e4/HGK-Mediathek. 